\title{
LE SESSE NELLE BAIE MARINE E LA QUESTIONE DELLA CORREZIONE DI BOCCA $\left(^{*}\right)$
}

\author{
Gerhard Neumann
}

I bacini d'acqua completamente chiusi; p. es. in laghi circondati da ogni parte da terra ferma, i periodi e la distribuzione di ampiezze delle sesse dipendono solo dalla lunghezza e dalle proporzioni di larghezza e profondità del bacino lacustre. In masse d'acqua non completamente delimitate, cioè in baie marine e in laghi che stanno in comunicazione col mare aperto mediante canali stretti o vie di mare, si aggiunge alla dipendenza della configurazione del bacino anche l'influsso delle ascillazioni libere ad opera della natura e della grandezza dell'apertura di foce.

Linflusso di aperture di qualsiasi forma e grandezza, in qualsivoglia punto del bacino sui periodi propri di tali masse d'acqua incompletamente chiuse, venne studiato dall'Autore (1) con il metodo della impedenza ${ }^{(2)}$ e i risultati furono comprovati con esperimenti e osservazioni su appropriati modelli di bacini. Il caso tanto frequente in natura, di una baia aperta sul lato stretto (fig. $1 c, d$, e) risulta dalle equazioni dei periodi come caso speciale del problema più generale di un bacino d'acqua aperto in qualsivoglia punto. Nella figura schematica 1 sono presentati semplici bacini rettangolari di profondita costante, solo per spiegare la posizione del problema. Ma il metodo si può applicare, come è stato indicato altrove (3), anche a bacini conformati irregolarmente di larghezza e profondità variabile.

Il lago rappresentato schematicamente nella fig. $1 b$, aperto lateralmente, costituisce un sistema oscillante composto, le cui singole parti si possono considerare come sistemi separati di oscillazione. Per effetto della loro azione simultanea ha luogo una oscillazione risultante del sistema complessivo, e noi ricerchiamo - neriodi propri di una tale massa d'acqua incompletamente delimitata.

Il complessivo spazio di oscillazione lo dividiamo in 4 campi (fig. 1 b). Il campo di oscillazione 1 viene computato dalla sezione trasversale nel punto dell'asse centrale dell'apertura di deflusso verso una estremità, il campo II verso l'altra estremità chiusa. Il campo III abbraccia il canale di sbocco ed il campo IV è lo spazio esterno nel campo aperto del lago.

Nel campo III si compie il passaggio dalle onde stazionarie piane alle onde progressive sferiche cilindriche dello spazio esterno o viceversa.

La teoria mostra che, aumentando la grandezza dell'apertura di sbocco e diminuendo la lunghezza del canale di sbocco, i periodi di sessa si fanno più piccoli in confronto ai periodi di un bacino completamente chiuso. Se l'apertura di bocca s'avvicina completamente ad una estremità del bacino, come ̀̀ rappresentato schematicamente nella fig. 1 , allora si hă

$$
\frac{\therefore}{c_{1}} \operatorname{tang} \omega \frac{I^{\prime}}{c_{1}}=\frac{a}{\omega} \quad \text { oppure } \frac{S_{1}}{c_{1}} \operatorname{tang} \pi \frac{T_{1}}{T}=\frac{a}{2 \pi} T,
$$

(*) Traduzione in lingua italiana della Nota di pag. 25. 
dove $T_{\imath}$ è il periodo del bacino che si pensa completamente chiuso (periodo non corretto), che può essere calcolato, per esempio con il metodo di Defant; $S_{1}=b_{1} h_{1}$ e $c_{1}=2 L_{1} / T_{1}$, se $b_{1}, h_{1}$ e $L_{1}$ indicano la larghezza, la profondità e la lunghezza (lungo la linea di valle! del lago; $T$ è il periodo (corretto), da determinarsi, del bacino lacustre aperto (baia), ed $a$ è una certa costante, che contiene le dimensioni dell'apertura di fase. Se questa ha la larghezza $b$, la profondità $h$ e l'(effettiva » lunghezza $l^{*}$, allora $a=b h / 1^{*}$ è la “capacità di conduzione " dell'apertura di bocca.

Le soluzioni dell'equazione trascendente [1] mostrano che per la presenza di un'apertura $\mathrm{i}$ periodi $T_{.}=2 L_{1} / n c_{1}, n=1,2,3, \ldots$ (oscillazione del lago) del bacino si abbassano ed aumentano $\mathrm{i}$ periodi $T_{\mathrm{b}}=2 L_{1} /\left(n+\frac{f}{5}\right) c_{1}, n=0,1,2,3, \ldots$ (oscillazione della baia) (2).

Aumentando la grandezza dell'apertura di bocca i periodi di risonanza del bacino si fanno continuamente più piccoli. Le oscillazioni di risonanza che appaiono, possono essere chiamate con egual diritto tanto "oscillazioni del lago abbreviate 》 od anche "oscillazioni allungate della baia".

Il cambiamento dei periodi di sessa dipende, dunque essenzialmente dalla “capacità di conduzione " a dell'apertura di bocca. Questa grandezza a contiene l'«effettiva » lunghezza $l^{*}=l+\alpha$ della bocea. $l$ ̀̀ la lunghezza geometrica della apertura di bocca e $\alpha$ un tratto aggiunto, la "correzione di bocca ".

Di speciale importanza è il caso in cui la sezione trasversale della foce $\grave{e}$ approssimativamente uguale alla sezione trasversale della baia. Allora la baia (fig. $1 l$ ) trapassa immediatamente nel campo aperto del mare e per l'ueffettiva " lunghezza della bocca si deve semplicemente porre la correzione di bocca. La grandezza di questa correzione si può determinare teoricamente sotto certe ipotesi, in relazione ad analoghe ricerche per le oscillazioni assiali dell'aria in tubi chiusi da un solo lato.

Nel caso di $S_{1}=b h=q$ la correzione di bocca diventa $l^{*}=\alpha$ e per $\alpha$ otteniamo, con buona approssimazione. $\alpha=0,347(b+h)$.

Dall'equazione [1] segue con $T_{1}=4 L_{1 /} c_{1}$ e $b h=q$

$$
\frac{\pi}{2}\left(1-\frac{T_{1}}{T}\right)=\operatorname{arctg} 2 \pi \frac{S_{1} l^{*}}{q c_{1} T} .
$$

e, dopo sviluppo dell'arctg, in prima approssimazione,

oppure

$$
1-\frac{T_{1}}{T} \approx \frac{4 S_{1} l^{*}}{q c_{1} T}
$$

$$
T=\frac{4\left(l_{1}+l^{*} S_{1} / q\right)}{c_{1}}=4 \frac{L}{c_{1}} \quad .
$$

$L^{\prime}=L_{1}+l^{*} . S_{1} / q$ è la lunghezza della baia “ridotta » o "effettiva ". Il periodo

(1) Della derivazione della equazione dei periodi e della applicazione del metodo, vedi le trattazioni originali (2) e (3).

(2) Nel caso dı un bacino aperto sul fianco, com"è rappresentato schematicamente nella fig. $1 b$, l'equazione del periodo sarebbe $\frac{S_{1}}{c_{1}} \operatorname{tang} \omega \frac{L_{1}}{c_{1}}+\frac{S_{2}}{c_{9}} \operatorname{tang}(1) \frac{L_{0}}{c_{2}}$ $=\frac{a}{w} \mathrm{da}$ cui segue l'equazione $[1]$ come caso speciale. 
ralcolato secondo questa formula di approssimazione sarà tanto più preciso, quanto più lunga è la baia in rapporto alla correzione $l^{*} S_{1} / q=(l+\alpha) S_{1} / q$. Trattandosi di baie corte, con apertura relativamente ampia, si calcola la durata corretta di oscillazione $T$ con più esattezza con l'equazione [1] o [2]. Dalla [3] deriva, con $l^{*}=l+0,374(b+h)$,

$$
\frac{T}{I_{1}}=1+\frac{l^{*}}{L_{1}} \frac{S_{1}}{q}=1+\frac{A}{a},
$$

se analogamente alla capacità di conduzione $n=q_{1} l^{*}$ designamo con $A$ il rapporto $S_{1} / L_{1}$ del bacino.

Mediante misurazioni di sesse prodotte artificialmente in appropriati bacini modello, con aperture diverse venne determinato sperimentalmente la dipendenza del fattore di correzione $T / T_{1}$ dalle dimensioni del bacino e della bocea $(A / a)$. 11 risultato di queste osservazioni è rappresentato nella fig. 2. La curva segnata darebbe per risultato il decorso della correzione secondo la "teoria giapponese " ${ }^{(t)}$. Vediamo che la formula usata finora nella oceanografia (v. Honda e collaboratori) (\$) per calcolare la correzione della foce dà per risultato valori, che si scostano notevolmente dalle osservazioni su modelli di bacini.

In particolari serie di esperimenti si studiò l'influsso di canali di sbocco, estesi in lunghezza, di diversa larghezza e profondita, sui periodi di sessa di masse d'acqua delimitate. Questi bacini modello avevano all'incirca una forma com'è rappresentato schematicamente nella fig. 1 . In luogo della lunghezza geometrica $l$ del canale, fu posta l'effettiva lunghezza del canale $l^{*}=l+0,374(b+h)$. Nella fig. 3 è rappresentata la dipendenza dei periodi propri dalla profondità $h$ dell'apertura di bocea per due diverse serie di esperimenti. La curva tracciata da' l'andamento secondo la formula [1] e i punti indicano i periodi osservati. La coincidenza fra teoria e osservazione è molto soddisfacente. Le dimensioni del bacino di esperimento e del canale di sbocco sono pure indicate nelle figure. L'abbassamento dei periodi $\dot{e}$ in coincidenza con la teoria tanto maggiormente, quanto maggiore è la sezione trasversale dell'apertura di bocca e quanto più breve è la lunghezza del canale di sbocco. Spargendo polvere d'alluminio, si poté inoltre rendere visibile la posizione dei ventri e dei nodi di oscillazione. Nella serie sperimentale $b$ ) nella fig. 3 vennero misurati anche i periodi della prima oscillazione superiore. Qui le osservazioni, bene coincidendo con la teoria, diedero per risultato una diminuzione del periodo proprio di molto più lieve, quando aumenta la grandezza della sezione trasversale della bocca.

\section{RIASSUNTO}

Viene investigato l'influsso di aperture, di qualsiasi formn ed estensione, comunque disposte nei riguardi del bacino, sui periodi propri di masse d'acqua incompletamente delimitate. Largomento è strettamente collegato al problema della correzione di bocca per sesse in golfi marini. La questione viene trattata teoricamente e sperimentalmente, in connessione con la "Impedanztheorie der Seiches 》. 